\title{
THE FINITE-TIME RUIN PROBABILITY WITH DEPENDENT INSURANCE AND FINANCIAL RISKS
}

\author{
YIQING CHEN,* University of Liverpool
}

\begin{abstract}
Consider a discrete-time insurance risk model. Within period $i$, the net insurance loss is denoted by a real-valued random variable $X_{i}$. The insurer makes both risk-free and risky investments, leading to an overall stochastic discount factor $Y_{i}$ from time $i$ to time $i-1$. Assume that $\left(X_{i}, Y_{i}\right), i \in \mathbb{N}$, form a sequence of independent and identically distributed random pairs following a common bivariate Farlie-Gumbel-Morgenstern distribution with marginal distribution functions $F$ and $G$. When $F$ is subexponential and $G$ fulfills some constraints in order for the product convolution of $F$ and $G$ to be subexponential too, we derive a general asymptotic formula for the finite-time ruin probability. Then, for special cases in which $F$ belongs to the Fréchet or Weibull maximum domain of attraction, we improve this general formula to be transparent.
\end{abstract}

Keywords: Asymptotics; Farlie-Gumbel-Morgenstern distribution; maximum domain of attraction; finite-time ruin probability; subexponential distribution

2010 Mathematics Subject Classification: Primary 62P05

Secondary 62E10; 91B30

\section{Introduction}

Following the works of Nyrhinen (1999), (2001) and Tang and Tsitsiashvili (2003), (2004), we consider a discrete-time insurance risk model. Within period $i$, the net insurance loss (equal to the total claim amount minus the total premium income) is denoted by a real-valued random variable $X_{i}, i \in \mathbb{N}$. Suppose that the insurer makes both risk-free and risky investments, which lead to an overall stochastic discount factor $Y_{i}$ from time $i$ to time $i-1$. See, e.g. Section 4.1 of Hashorva et al. (2010) for the structure of these overall stochastic discount factors. In the terminology of Tang and Tsitsiashvili (2003), (2004), we call $\left\{X_{i}, i \in \mathbb{N}\right\}$ insurance risks and $\left\{Y_{i}, i \in \mathbb{N}\right\}$ financial risks. Thus, the sum

$$
S_{n}=\sum_{i=1}^{n} X_{i} \prod_{j=1}^{i} Y_{j}, \quad n \in \mathbb{N},
$$

represents the stochastic present value of aggregate net losses up to time $n$. As usual, the probability of ruin by time $n$ is defined to be

$$
\psi(x ; n)=\operatorname{Pr}\left(\max _{1 \leq m \leq n} \sum_{i=1}^{m} X_{i} \prod_{j=1}^{i} Y_{j}>x\right), \quad n \in \mathbb{N},
$$

where $x \geq 0$ is interpreted as the initial wealth of the insurer.

Received 10 May 2011; revision received 13 July 2011.

* Postal address: Department of Mathematical Sciences, University of Liverpool, Liverpool, L69 7ZL, UK.

Email address: yiqing.chen@liv.ac.uk 
Since the work of Tang and Tsitsiashvili (2003), there has been a vast amount of literature on the asymptotic behavior of the ruin probability of this risk model in the presence of heavytailed insurance and/or financial risks. It has been common to assume that both $\left\{X_{i}, i \in \mathbb{N}\right\}$ and $\left\{Y_{i}, i \in \mathbb{N}\right\}$ are sequences of independent and identically distributed (i.i.d.) random variables and that they are independent of each other. Undoubtedly, this assumption of complete independence is unrealistic. A recent new approach is to introduce various dependence structures to the risk model. In this direction, we refer the reader to Goovaerts et al. (2005), Tang (2006a), Zhang et al. (2009), Weng et al. (2009), and Yi et al. (2011), among many others. However, there are few papers which take into account the dependence between insurance and financial risks, with the difficulty existing in describing the tail behavior of the product of dependent random variables.

In the present paper we assume that $\left(X_{i}, Y_{i}\right), i \in \mathbb{N}$, form a sequence of i.i.d. random pairs, and that a generic random pair $(X, Y)$ has dependent components. We use a bivariate FarlieGumbel-Morgenstern (FGM) distribution to model the dependence structure of $(X, Y)$. Recall that a bivariate FGM distribution function is of the form

$$
\Pi(x, y)=F(x) G(y)(1+\theta \bar{F}(x) \bar{G}(y)),
$$

where $F$ and $G$ are marginal distribution functions and $\theta$ is a real number fulfilling $|\theta| \leq 1$ in order for $\Pi(\cdot, \cdot)$ to be a proper bivariate distribution function. Trivially, if $\theta=0$ then (1.3) describes a joint distribution function of two independent random variables. We refer the reader to Kotz et al. (2000) for a general account on FGM distribution functions, and to Tang and Vernic (2007) and Cossette et al. (2008) for applications of FGM distribution functions to risk theory.

First, under the assumption that $F$ in (1.3) is a subexponential distribution function while $G$ fulfills some constraints in order for the product convolution of $F$ and $G$ to be a subexponential distribution function too, we derive a general asymptotic formula for the ruin probability $\psi(x ; n)$. Here the product convolution of $F$ and $G$, denoted as $F \otimes G$, is understood as the distribution function of the product $X^{*} Y^{*}$, where $X^{*}$ and $Y^{*}$ are two independent random variables with $X^{*}$ identically distributed as $X$ and $Y^{*}$ as $Y$.

Next, for some special cases in which $F$ is a distribution function in the Fréchet or Weibull maximum domain of attraction, we improve the general asymptotic formula to be transparent. The obtained asymptotic formulae successfully capture the impact of the underlying dependence structure of $(X, Y)$.

The rest of this paper consists of three sections. In Section 2 we give some preliminaries on subexponential distributions and maximum domains of attraction. Section 3 contains the main results, and the proofs are presented in Section 4.

\section{Preliminaries}

Throughout the paper, all limit relationships are according to $x \rightarrow \infty$ unless otherwise stated. For two positive functions $f(\cdot)$ and $g(\cdot)$, we write $f(x) \lesssim g(x)$ or $g(x) \gtrsim f(x)$ if $\limsup f(x) / g(x) \leq 1$ and write $f(x) \sim g(x)$ if $\lim f(x) / g(x)=1$. We also write $f(x) \asymp g(x)$ if $0<\liminf f(x) / g(x) \leq \lim \sup f(x) / g(x)<\infty$.

A distribution function $F$ on $\mathbb{R}^{+}=[0, \infty)$ is said to be subexponential, written as $F \in \&$, if $\bar{F}(x)=1-F(x)>0$ for all $x \in \mathbb{R}^{+}$and

$$
\overline{F^{2 *}}(x) \sim 2 \bar{F}(x)
$$


where $F^{2 *}$ denotes the two-fold convolution of $F$. More generally, a distribution function $F$ on $\mathbb{R}$ is still said to be subexponential if the distribution function $F_{+}(x)=F(x) \mathbf{1}_{\{x \geq 0\}}$ is subexponential. It is well known that every subexponential distribution function $F$ is long tailed, written as $F \in \mathcal{L}$, in the sense that the relation $\bar{F}(x+y) \sim \bar{F}(x)$ holds for some (or, equivalently, for all) $y \neq 0$; see Lemma 1.3.5(a) of Embrechts et al. (1997). Following the works of Konstantinides et al. (2002) and Tang (2006b), we say that a distribution function $F$ on $\mathbb{R}$ belongs to the class $\mathcal{A}$ if $F \in \&$ and the relation

$$
\limsup _{x \rightarrow \infty} \frac{\bar{F}(c x)}{\bar{F}(x)}<1
$$

holds for some $c>1$. Note that (2.1) is really a mild constraint and is fulfilled by almost all useful distribution functions with unbounded supports. Thus, as remarked in Tang (2006b), the class $\mathcal{A}$ almost exhausts the class $\&$.

Under the assumption that the insurance risk $X$ follows a distribution function $F \in \delta$ or $\mathcal{A}$, we will derive a general asymptotic formula for the ruin probability $\psi(x ; n)$. In order to further improve this general formula to be transparent, we need to introduce several concepts in extreme value theory.

A distribution function $F$ on $\mathbb{R}$ is said to belong to the maximum domain of attraction of an extreme value distribution function $F_{0}$, written as $F \in \operatorname{MDA}\left(F_{0}\right)$, if

$$
\lim _{n \rightarrow \infty} \sup _{x \in \mathbb{R}}\left|F^{n}\left(a_{n} x+b_{n}\right)-F_{0}(x)\right|=0
$$

holds for some constants $a_{n}>0$ and $b_{n} \in \mathbb{R}, n \in \mathbb{N}$. Only three choices for $F_{0}$ in (2.2) are possible: the Fréchet, Gumbel, and Weibull distributions, which we denote by $\Phi_{\alpha}, \Lambda$, and $\Psi_{\alpha}$ for $\alpha>0$, respectively. A distribution function $F$ belongs to $\operatorname{MDA}\left(\Phi_{\alpha}\right)$ if and only if $\bar{F}(\cdot)$ is regularly varying at $\infty$ with index $-\alpha$, that is, the relation

$$
\lim _{x \rightarrow \infty} \frac{\bar{F}(c x)}{\bar{F}(x)}=c^{-\alpha}
$$

holds for all $c>0$; see Theorem 3.3.7 of Embrechts et al. (1997). Hence, $\operatorname{MDA}\left(\Phi_{\alpha}\right) \subset \&$; see Lemma 1.3.1 of Embrechts et al. (1997). A distribution function $F$ with an upper endpoint $\hat{x}=\sup \{x \in \mathbb{R}: F(x)<1\} \leq \infty$ belongs to $\operatorname{MDA}(\Lambda)$ if and only if the relation

$$
\lim _{x \uparrow \hat{x}} \frac{\bar{F}(x+c a(x))}{\bar{F}(x)}=\mathrm{e}^{-c}
$$

holds for some positive auxiliary function $a(\cdot)$ and all $c \in \mathbb{R}$. Recall that the auxiliary function $a(\cdot)$ is asymptotically equivalent to the mean excess function of $F$, namely, $a(x) \sim \mathrm{E}[X-$ $x \mid X>x]$ as $x \uparrow \hat{x}$; see Theorem 3.3.27 of Embrechts et al. (1997). Almost all distribution functions with rapidly varying tails are included in $\operatorname{MDA}(\Lambda)$. A distribution function $F$ belongs to $\operatorname{MDA}\left(\Psi_{\alpha}\right)$ if and only if its upper endpoint $\hat{x}$ is finite and the relation

$$
\lim _{x \rightarrow \infty} \frac{\bar{F}(\hat{x}-c / x)}{\bar{F}(\hat{x}-1 / x)}=c^{\alpha}
$$

holds for all $c>0$; see Theorem 3.3.12 of Embrechts et al. (1997). 


\section{Main results}

Recall the insurance risk model introduced in Section 1 . In the sequel, denote by $F$ on $\mathbb{R}$, $G$ on $\mathbb{R}^{+}$, and $H$ on $\mathbb{R}^{+}$the distribution functions of $X, Y$, and $X Y$, respectively. Throughout the rest of the paper, $F$ is assumed to be heavy tailed and, to avoid triviality, $G$ is assumed to be nondegenerate at 0 . For each $i \in \mathbb{N}$, denote by $H_{i}$ the distribution function of $X_{i} \prod_{j=1}^{i} Y_{j}$. Note that $H=H_{1}$.

Our first main result is as follows.

Theorem 3.1. Assume that $\left(X_{i}, Y_{i}\right), i \in \mathbb{N}$, form a sequence of i.i.d. random pairs following a common bivariate FGM distribution function (1.3) with $\theta \in(-1,1]$. Furthermore, assume that $F \in \&$ and that there is a function $b(\cdot): \mathbb{R}^{+} \mapsto \mathbb{R}^{+}$such that the following statements hold simultaneously:

(a) $b(x) \uparrow \infty$,

(b) $b(x) / x \downarrow 0$,

(c) $\bar{G}(b(x))=o(\bar{H}(x))$, and

(d) $\bar{F}(x-b(x)) \sim \bar{F}(x)$.

Then, for each $n \in \mathbb{N}$, the distribution function of $S_{n}$ belongs to the class \& and the ruin probability $\psi(x ; n)$ satisfies

$$
\psi(x ; n) \sim \operatorname{Pr}\left(S_{n}>x\right) \sim \sum_{i=1}^{n} \bar{H}_{i}(x) .
$$

Assumptions (a)-(d) of Theorem 3.1 were first proposed in Cline and Samorodnitsky (1994) when establishing subexponentiality for the product of independent random variables; see Lemma 4.1 below. These assumptions automatically hold if $Y$ is bounded above. Recall that $F \otimes G$ stands for the product convolution of $F$ and $G$. With $\theta \in(-1,1]$, relations (4.4) and (4.7) below show that $\bar{H}(x) \asymp \overline{F \otimes G}(x)$. Thus, for this case, the set of assumptions (a)-(c) is equivalent to the set of assumptions (a), (b), and

(c*) $\bar{G}(b(x))=o(\overline{F \otimes G}(x))$.

Of course, $\left(\mathrm{c}^{*}\right)$ is easier to verify than (c).

As remarked in Tang (2006b), (c) requires that $b(\cdot)$ diverge to $\infty$ not too slowly while (d) requires that it diverge to $\infty$ not too fast. Very often (d) appears to be too restrictive for applications. In our second main result below we drop this annoying assumption.

Theorem 3.2. Assume that $\left(X_{i}, Y_{i}\right), i \in \mathbb{N}$, form a sequence of i.i.d. random pairs following a common bivariate FGM distribution function (1.3) with $\theta \in(-1,1]$. Furthermore, assume that $F \in \mathcal{A}$ and that there is a function $b(\cdot): \mathbb{R}^{+} \mapsto \mathbb{R}^{+}$such that (a)-(c) hold simultaneously. Then, for each $n \in \mathbb{N}$, the distribution function of $S_{n}$ belongs to the class $\mathcal{A}$ and the ruin probability $\psi(x ; n)$ satisfies $(3.1)$.

Interestingly, as summarized in Lemma 4.6 below, (a)-(c) amount to the assertion that the relation $\bar{G}(c x)=o(\bar{H}(x))$ holds for all $c>0$. This latter assertion is much easier to verify than (a)-(c). 
We end this section with a corollary of Theorems 3.1 and 3.2 in which we focus on improving the asymptotic formula given in (3.1) to be completely transparent within maximum domains of attraction.

Corollary 3.1. Assume that $\left(X_{i}, Y_{i}\right), i \in \mathbb{N}$, form a sequence of i.i.d. random pairs following a common bivariate FGM distribution function (1.3) with $\theta \in(-1,1]$.

(i) If $F \in \operatorname{MDA}\left(\Phi_{\alpha}\right)$ for some $\alpha>0$ and $\mathrm{E}\left[Y^{\alpha+\varepsilon}\right]<\infty$ for some $\varepsilon>0$, then

$$
\psi(x ; n) \sim\left((1-\theta) \mathrm{E}\left[Y^{\alpha}\right]+\theta \mathrm{E}\left[\left(Y_{1}^{*} \vee Y_{2}^{*}\right)^{\alpha}\right]\right) \frac{1-\left(\mathrm{E}\left[Y^{\alpha}\right]\right)^{n}}{1-\mathrm{E}\left[Y^{\alpha}\right]} \bar{F}(x),
$$

where $Y_{1}^{*}$ and $Y_{2}^{*}$ are two independent random variables identically distributed as $Y$.

(ii) If $F \in \& \cap \operatorname{MDA}(\Lambda)$ with an auxiliary function $a(\cdot)$ and $G \in \operatorname{MDA}\left(\Psi_{\alpha}\right)$ for some $\alpha>0$ with an upper endpoint $0<\hat{y}<\infty$, then

$$
\psi(x ; n) \sim(1+\theta) \sum_{i=1}^{n} \bar{F}\left(\frac{x}{\hat{y}^{i}}\right)\left(\Gamma(\alpha+1) \bar{G}\left(\hat{y}-\frac{\hat{y}^{i+1}}{x} a\left(\frac{x}{\hat{y}^{i}}\right)\right)\right)^{i} .
$$

Relation (3.2) suggests that if further $\mathrm{E}\left[Y^{\alpha}\right]<1$, the following simple asymptotic formula for the infinite-time ruin probability should hold:

$$
\psi(x ; \infty)=\operatorname{Pr}\left(\max _{1 \leq m<\infty} \sum_{i=1}^{m} X_{i} \prod_{j=1}^{i} Y_{j}>x\right) \sim \frac{(1-\theta) \mathrm{E}\left[Y^{\alpha}\right]+\theta \mathrm{E}\left[\left(Y_{1}^{*} \vee Y_{2}^{*}\right)^{\alpha}\right]}{1-\mathrm{E}\left[Y^{\alpha}\right]} \bar{F}(x) .
$$

Indeed, this can be easily proven by Theorem 1 of Grey (1994). We omit the details. Also, note that, when $\hat{y}=1$, relation (3.3) admits the following substantial simplification:

$$
\psi(x ; n) \sim(1+\theta) \Gamma(\alpha+1) \bar{F}(x) \bar{G}\left(1-\frac{a(x)}{x}\right) .
$$

Since the right-hand side of (3.4) does not involve $n$, the same asymptotic formula should hold for the infinite-time ruin probability, though we still cannot prove it at this stage.

\section{Proofs}

Recall relations (1.1) and (1.2). Clearly,

$$
\operatorname{Pr}\left(\sum_{i=1}^{n} X_{i} \prod_{j=1}^{i} Y_{j}>x\right) \leq \psi(x ; n) \leq \operatorname{Pr}\left(\sum_{i=1}^{n} X_{i}^{+} \prod_{j=1}^{i} Y_{j}>x\right),
$$

where $X_{i}^{+}=X_{i} \mathbf{1}_{\left\{X_{i} \geq 0\right\}}$ denotes the positive part of $X_{i}, i \in \mathbb{N}$. For the proof that (3.1) holds in both Theorems 3.1 and 3.2, if we can establish that the second asymptotic formula in (3.1) holds by not requiring the assumption that $F(0-)>0$, then the same asymptotic formula should hold for the right-hand side of (4.1) as well. In this way we will have completed the proof of (3.1). Furthermore, we note the simple fact that

$$
S_{n}=\sum_{i=1}^{n} X_{i} \prod_{j=1}^{i} Y_{j} \stackrel{\mathrm{D}}{=} \sum_{i=1}^{n} X_{i} \prod_{j=i}^{n} Y_{j}, \quad n \in \mathbb{N},
$$


due to the i.i.d. assumption for the sequence $\left\{\left(X_{i}, Y_{i}\right), i \in \mathbb{N}\right\}$, where ' $=$, denotes equality in distribution. Denote by $T_{n}$ the sum on the right-hand side of (4.2). Therefore, we only need to prove the relation

$$
\operatorname{Pr}\left(T_{n}>x\right) \sim \sum_{i=1}^{n} \bar{H}_{i}(x) .
$$

\subsection{Proof of Theorem 3.1}

The following lemma is a restatement of Theorem 2.1 of Cline and Samorodnitsky (1994), which is crucial for establishing our Theorem 3.1.

Lemma 4.1. Let $X$ and $Y$ be two independent random variables with distribution functions $F$ on $\mathbb{R}$ and $G$ on $\mathbb{R}^{+}$, respectively. Denote by $H$ the distribution function of their product $X Y$. We have $H \in \delta$ if $F \in$ s and there is a function $b(\cdot): \mathbb{R}^{+} \mapsto \mathbb{R}^{+}$such that $(a)-(d)$, namely,

- $b(x) \uparrow \infty$,

- $b(x) / x \downarrow 0$,

- $\bar{G}(b(x))=o(\bar{H}(x))$, and

- $\bar{F}(x-b(x)) \sim \bar{F}(x)$

hold simultaneously.

Recall the generic random pair $(X, Y)$ in the insurance risk model in Section 1. Introduce the six independent random variables $X^{*}, X_{1}^{*}, X_{2}^{*}, Y^{*}, Y_{1}^{*}$, and $Y_{2}^{*}$, with the first three identically distributed as $X$ and the last three identically distributed as $Y$. In the proof of Theorem 3.1 we also need the following lemma.

Lemma 4.2. Let $(X, Y)$ follow a bivariate $F G M$ distribution function $(1.3)$ with $\theta \in(-1,1]$. Under (a)-(c), it holds that

$$
\bar{H}(x) \sim(1-\theta) \operatorname{Pr}\left(X^{*} Y^{*}>x\right)+\theta \operatorname{Pr}\left(X^{*}\left(Y_{1}^{*} \vee Y_{2}^{*}\right)>x\right) .
$$

Proof. Note the decomposition

$$
\Pi=(1+\theta) F G-\theta F^{2} G-\theta F G^{2}+\theta F^{2} G^{2} .
$$

Clearly, $X_{1}^{*} \vee X_{2}^{*}$ is distributed by $F^{2}$ and $Y_{1}^{*} \vee Y_{2}^{*}$ is distributed by $G^{2}$. Thus, it follows from (4.5) that

$$
\begin{aligned}
\bar{H}(x)= & \operatorname{Pr}(X Y>x) \\
= & (1+\theta) \operatorname{Pr}\left(X^{*} Y^{*}>x\right)-\theta \operatorname{Pr}\left(\left(X_{1}^{*} \vee X_{2}^{*}\right) Y^{*}>x\right) \\
& -\theta \operatorname{Pr}\left(X^{*}\left(Y_{1}^{*} \vee Y_{2}^{*}\right)>x\right)+\theta \operatorname{Pr}\left(\left(X_{1}^{*} \vee X_{2}^{*}\right)\left(Y_{1}^{*} \vee Y_{2}^{*}\right)>x\right) .
\end{aligned}
$$

In terms of the function $b(\cdot)$ in (a)-(c), we have

$$
\begin{aligned}
\operatorname{Pr}\left(\left(X_{1}^{*} \vee X_{2}^{*}\right) Y^{*}>x\right) & =\int_{0}^{b(x)} \operatorname{Pr}\left(X_{1}^{*} \vee X_{2}^{*}>\frac{x}{y}\right) \mathrm{d} G(y)+O(\bar{G}(b(x))) \\
& =(2+o(1)) \int_{0}^{b(x)} \operatorname{Pr}\left(X^{*}>\frac{x}{y}\right) \mathrm{d} G(y)+O(\bar{G}(b(x))) \\
& =(2+o(1)) \operatorname{Pr}\left(X^{*} Y^{*}>x\right)+o(\bar{H}(x)) .
\end{aligned}
$$


Similarly as above,

$$
\operatorname{Pr}\left(\left(X_{1}^{*} \vee X_{2}^{*}\right)\left(Y_{1}^{*} \vee Y_{2}^{*}\right)>x\right)=(2+o(1)) \operatorname{Pr}\left(X^{*}\left(Y_{1}^{*} \vee Y_{2}^{*}\right)>x\right)+o(\bar{H}(x))
$$

Substituting these estimates into (4.6) yields

$$
\bar{H}(x)=(1-\theta+o(1)) \operatorname{Pr}\left(X^{*} Y^{*}>x\right)+(\theta+o(1)) \operatorname{Pr}\left(X^{*}\left(Y_{1}^{*} \vee Y_{2}^{*}\right)>x\right)+o(\bar{H}(x)),
$$

which implies relation (4.4).

When $\theta \in(-1,1]$, we apply the two-sided inequality

$$
\operatorname{Pr}\left(X^{*} Y^{*}>x\right) \leq \operatorname{Pr}\left(X^{*}\left(Y_{1}^{*} \vee Y_{2}^{*}\right)>x\right) \leq 2 \operatorname{Pr}\left(X^{*} Y^{*}>x\right)
$$

to obtain an asymptotic estimate for the right-hand side of (4.4) as

$$
(1-\theta) \operatorname{Pr}\left(X^{*} Y^{*}>x\right)+\theta \operatorname{Pr}\left(X^{*}\left(Y_{1}^{*} \vee Y_{2}^{*}\right)>x\right) \asymp \operatorname{Pr}\left(X^{*} Y^{*}>x\right) .
$$

Therefore, there is no problem with the implication in the last step of the proof of Lemma 4.2. When $\theta=-1$, however, we need to be aware that the right-hand side of (4.4) could reduce to $o\left(\operatorname{Pr}\left(X^{*} Y^{*}>x\right)\right)$, e.g. when $F$ is rapidly varying tailed and $G$ does not assign a mass at the upper endpoint $0<\hat{y} \leq \infty$. For this case, (4.4) may fail to give an exact asymptotic relationship. This explains why we have to exclude $\theta=-1$ in Lemma 4.2 and hereby in Theorems 3.1 and 3.2.

Lemma 4.2 offers the following insight. For $\theta \in(-1,1]$, introduce a function $G_{\theta}(\cdot): \mathbb{R}^{+} \mapsto$ $\mathbb{R}^{+}$as

$$
G_{\theta}(y)=(1-\theta) G(y)+\theta G^{2}(y),
$$

which clearly defines a proper distribution function on $\mathbb{R}^{+}$. Furthermore, introduce a nonnegative random variable $Z_{\theta}$ distributed according to $G_{\theta}$ and independent of $(X, Y)$. Then relation (4.4) can be rewritten as

$$
\operatorname{Pr}(X Y>x) \sim \operatorname{Pr}\left(X Z_{\theta}>x\right) .
$$

In this way, the dependence structure of $(X, Y)$ is dissolved.

By Lemma 4.1 , it is easy to establish the subexponentiality of the independent product $X Z_{\theta}$ in (4.9). Also, recall that the class $\delta$ is closed under asymptotic equivalence; see, e.g. Theorem 3 of Teugels (1975). Therefore, by relation (4.9) we readily arrive at the following result.

Lemma 4.3. Let $(X, Y)$ follow a bivariate FGM distribution function (1.3) with $\theta \in(-1,1]$. If $F \in \&$ and $(a)-(d)$ hold then $H \in \&$.

For a distribution function $F$ on $\mathbb{R}$, under (a), (b), and (d), it is straightforward to verify that the relation

$$
\bar{F}(x-c b(x)) \sim \bar{F}(x)
$$

holds for all $c>0$. The following lemma is inspired by the work of Zhou et al. (2011).

Lemma 4.4. Let $(X, Y)$ follow a bivariate FGM distribution function (1.3) with $\theta \in(-1,1]$. Under $(a)-(d)$, it holds for all $c>0$ that

$$
\bar{H}(x-c b(x)) \sim \bar{H}(x) .
$$


Proof. We only need to prove that (4.11) holds with $c=1$. For arbitrarily fixed $0<\varepsilon, \delta<1$, by (4.9) we have

$$
\begin{aligned}
\bar{H}(x-b(x)) & \sim \operatorname{Pr}\left(X Z_{\theta}>x-b(x)\right) \\
& =\left(\int_{0}^{\varepsilon}+\int_{\varepsilon}^{1}+\int_{1}^{b(x)}+\int_{b(x)}^{\infty}\right) \bar{F}\left(\frac{x-b(x)}{y}\right) \mathrm{d} G_{\theta}(y) \\
& =\sum_{i=1}^{4} I_{i}(x) .
\end{aligned}
$$

We deal with the four terms on the right-hand side of (4.12) one by one. By (a) and relation (4.10) with $c=1 / \varepsilon$, it holds for all large $x$ that

$$
\begin{aligned}
I_{1}(x) & \leq \bar{F}\left(\frac{x-b(x)}{\varepsilon}\right) G_{\theta}((0, \varepsilon]) \\
& \leq \bar{F}\left(\frac{x}{\varepsilon}-\frac{1}{\varepsilon} b\left(\frac{x}{\varepsilon}\right)\right) G_{\theta}((0, \varepsilon]) \\
& \leq(1+\delta) G_{\theta}((0, \varepsilon]) \bar{F}\left(\frac{x}{\varepsilon}\right) \\
& =(1+\delta) \frac{G_{\theta}((0, \varepsilon])}{\overline{G_{\theta}}(\varepsilon)} \operatorname{Pr}\left(X>\frac{x}{\varepsilon}, Z_{\theta}>\varepsilon\right) \\
& \leq(1+\delta) \frac{G_{\theta}((0, \varepsilon])}{\overline{G_{\theta}}(\varepsilon)} \operatorname{Pr}\left(X Z_{\theta}>x\right) .
\end{aligned}
$$

Similarly, it holds for all large $x$ that

$$
\begin{aligned}
I_{2}(x) & \leq \int_{\varepsilon}^{1} \bar{F}\left(\frac{x}{y}-\frac{b(x)}{\varepsilon}\right) \mathrm{d} G_{\theta}(y) \\
& \leq \int_{\varepsilon}^{1} \bar{F}\left(\frac{x}{y}-\frac{1}{\varepsilon} b\left(\frac{x}{y}\right)\right) \mathrm{d} G_{\theta}(y) \\
& \leq(1+\delta) \int_{\varepsilon}^{1} \bar{F}\left(\frac{x}{y}\right) \mathrm{d} G_{\theta}(y) .
\end{aligned}
$$

By (b) and (d), it holds for all large $x$ that

$$
I_{3}(x) \leq \int_{1}^{b(x)} \bar{F}\left(\frac{x}{y}-b\left(\frac{x}{y}\right)\right) \mathrm{d} G_{\theta}(y) \leq(1+\delta) \int_{1}^{b(x)} \bar{F}\left(\frac{x}{y}\right) \mathrm{d} G_{\theta}(y) .
$$

Finally, by (4.8) and (c),

$$
I_{4}(x) \leq \bar{G}_{\theta}(b(x)) \asymp \bar{G}(b(x))=o(\bar{H}(x)) .
$$

Substituting these estimates into (4.12) yields

$$
\begin{aligned}
\bar{H}(x & -b(x)) \\
& \lesssim(1+\delta) \frac{G_{\theta}((0, \varepsilon])}{\bar{G}_{\theta}(\varepsilon)} \operatorname{Pr}\left(X Z_{\theta}>x\right)+(1+\delta) \int_{\varepsilon}^{b(x)} \bar{F}\left(\frac{x}{y}\right) \mathrm{d} G_{\theta}(y)+o(\bar{H}(x))
\end{aligned}
$$




$$
\begin{aligned}
& \lesssim(1+\delta)\left(\frac{G_{\theta}((0, \varepsilon])}{\bar{G}_{\theta}(\varepsilon)}+1\right) \operatorname{Pr}\left(X Z_{\theta}>x\right)+o(\bar{H}(x)) \\
& \sim(1+\delta)\left(\frac{G_{\theta}((0, \varepsilon])}{\bar{G}_{\theta}(\varepsilon)}+1\right) \bar{H}(x),
\end{aligned}
$$

where in the last step we used (4.9) again. Letting both $\varepsilon \downarrow 0$ and $\delta \downarrow 0$ on the right-hand side above leads to $\bar{H}(x-b(x)) \lesssim \bar{H}(x)$, which is equivalent to (4.11) with $c=1$.

The following lemma is well known and can be found in Embrechts and Goldie (1980), Cline (1986, Corollary 1), and Tang and Tsitsiashvili (2003, Lemma 3.2).

Lemma 4.5. Let $F_{1}$ and $F_{2}$ be two distribution functions on $\mathbb{R}$, and let $F=F_{1} * F_{2}$. If $F_{1} \in \delta$, $F_{2} \in \mathcal{L}$, and $\bar{F}_{2}(x)=O\left(\bar{F}_{1}(x)\right)$, then $F \in \&$ and $\bar{F}(x) \sim \bar{F}_{1}(x)+\bar{F}_{2}(x)$.

Proof of Theorem 3.1. As mentioned in the beginning of this section, we only need to prove that relation (4.3) holds and that the distribution function of $T_{n}$ belongs to the class $\delta$. We proceed by induction on $n$. Note that

$(1: 1) \bar{G}(b(x))=o(\bar{H}(x))$ and $\bar{H}(x-b(x)) \sim \bar{H}(x)$ (by Lemma 4.4),

(1:2) relation (4.3) trivially holds for $n=1$,

(1:3) $T_{1}=X_{1} Y_{1}$ follows a subexponential distribution function (by Lemma 4.3).

Now assume that

(n:1) $\bar{G}(b(x))=o\left(\bar{H}_{n}(x)\right)$ and $\bar{H}_{n}(x-b(x)) \sim \bar{H}_{n}(x)$,

(n:2) relation (4.3) holds for $n$,

(n:3) both $X_{n} \prod_{j=1}^{n} Y_{j}$ and $T_{n}$ follow subexponential distribution functions.

We will prove that these assertions hold for $n+1$, namely,

$(\mathrm{n}+1: 1) \bar{G}(b(x))=o\left(\bar{H}_{n+1}(x)\right)$ and $\bar{H}_{n+1}(x-b(x)) \sim \bar{H}_{n+1}(x)$,

$(\mathrm{n}+1: 2)$ relation $(4.3)$ holds for $n+1$,

$(\mathrm{n}+1: 3)$ both $X_{n+1} \prod_{j=1}^{n+1} Y_{j}$ and $T_{n+1}$ follow subexponential distribution functions.

First, note that $T_{n}$ and $X_{n+1}$ are independent of each other, that, by (n:3), both follow subexponential distribution functions (hence are long tailed), and that, by (n:2), if $\bar{G}(1)>0$ then $\operatorname{Pr}\left(X_{n+1}>x\right)=O\left(\operatorname{Pr}\left(T_{n}>x\right)\right)$, while if $\bar{G}(1)=0$ then $\operatorname{Pr}\left(T_{n}>x\right)=O\left(\operatorname{Pr}\left(X_{n+1}>x\right)\right)$. Thus, by Lemma 4.5 ,

$$
\operatorname{Pr}\left(T_{n}+X_{n+1}>x\right) \sim \operatorname{Pr}\left(T_{n}>x\right)+\operatorname{Pr}\left(X_{n+1}>x\right) \sim \sum_{i=1}^{n} \bar{H}_{i}(x)+\bar{F}(x),
$$

where in the last step we used (n:2).

Consider $(\mathrm{n}+1: 1)$. If $\bar{G}(1)>0$ then

$$
\bar{H}_{n+1}(x) \geq \operatorname{Pr}\left(X_{n+1} \prod_{j=1}^{n+1} Y_{j}>x, Y_{1}>1, \ldots, Y_{n}>1\right) \geq \bar{H}(x) \bar{G}(1)^{n} .
$$

Thus, $\bar{G}(b(x))=o(\bar{H}(x))=o\left(\bar{H}_{n+1}(x)\right)$. If $\bar{G}(1)=0$ then the relation $\bar{G}(b(x))=$ $o\left(\bar{H}_{n+1}(x)\right)$ trivially holds since $\bar{G}(b(x))=0$ for all large $x$. Moreover, note that, since 
$\bar{H}_{n}(x-b(x)) \sim \bar{H}_{n}(x)$ by $(\mathrm{n}: 1)$, the proof of $\bar{H}_{n+1}(x-b(x)) \sim \bar{H}_{n+1}(x)$ is similar to but easier than the proof of Lemma 4.4.

Next, consider $(\mathrm{n}+1: 2)$. Introduce the six independent random variables $X^{*}, X_{1}^{*}, X_{2}^{*}, Y^{*}$, $Y_{1}^{*}$, and $Y_{2}^{*}$, as for Lemma 4.2, and let them be independent of $T_{n}$. Similarly as done in (4.6),

$$
\begin{aligned}
\operatorname{Pr}\left(T_{n+1}>x\right)= & \operatorname{Pr}\left(\left(T_{n}+X_{n+1}\right) Y_{n+1}>x\right) \\
= & (1+\theta) \operatorname{Pr}\left(\left(T_{n}+X^{*}\right) Y^{*}>x\right)-\theta \operatorname{Pr}\left(\left(T_{n}+X_{1}^{*} \vee X_{2}^{*}\right) Y^{*}>x\right) \\
& -\theta \operatorname{Pr}\left(\left(T_{n}+X^{*}\right)\left(Y_{1}^{*} \vee Y_{2}^{*}\right)>x\right)+\theta \operatorname{Pr}\left(\left(T_{n}+X_{1}^{*} \vee X_{2}^{*}\right)\left(Y_{1}^{*} \vee Y_{2}^{*}\right)>x\right) \\
= & (1+\theta) J_{1}(x)-\theta J_{2}(x)-\theta J_{3}(x)+\theta J_{4}(x) .
\end{aligned}
$$

We only deal with the tail probability $J_{1}(x)$ in detail. By (4.13), for $x>0$,

$$
\begin{aligned}
J_{1}(x) & =\left(\int_{0}^{b(x)}+\int_{b(x)}^{\infty}\right) \operatorname{Pr}\left(T_{n}+X^{*}>\frac{x}{y}\right) \mathrm{d} G(y) \\
& =(1+o(1)) \int_{0}^{b(x)}\left(\sum_{i=1}^{n} \bar{H}_{i}\left(\frac{x}{y}\right)+\bar{F}\left(\frac{x}{y}\right)\right) \mathrm{d} G(y)+O(\bar{G}(b(x))) \\
& \sim \int_{0}^{\infty}\left(\sum_{i=1}^{n} \bar{H}_{i}\left(\frac{x}{y}\right)+\bar{F}\left(\frac{x}{y}\right)\right) \mathrm{d} G(y),
\end{aligned}
$$

where we have used $\bar{G}(b(x))=o(1) \int_{0}^{\infty} \bar{F}(x / y) \mathrm{d} G(y)$ due to (c) and relations (4.4) and (4.7). In a similar way, with some obvious modifications, we have

$$
\begin{aligned}
& J_{2}(x) \sim \int_{0}^{\infty}\left(\sum_{i=1}^{n} \bar{H}_{i}\left(\frac{x}{y}\right)+2 \bar{F}\left(\frac{x}{y}\right)\right) \mathrm{d} G(y), \\
& J_{3}(x) \sim \int_{0}^{\infty}\left(\sum_{i=1}^{n} \bar{H}_{i}\left(\frac{x}{y}\right)+\bar{F}\left(\frac{x}{y}\right)\right) \mathrm{d} G^{2}(y), \\
& J_{4}(x) \sim \int_{0}^{\infty}\left(\sum_{i=1}^{n} \bar{H}_{i}\left(\frac{x}{y}\right)+2 \bar{F}\left(\frac{x}{y}\right)\right) \mathrm{d} G^{2}(y) .
\end{aligned}
$$

Substituting these estimates into (4.14), and then using (4.8) and (4.9), we obtain

$$
\begin{aligned}
\operatorname{Pr}\left(T_{n+1}>x\right) \sim & \int_{0}^{\infty} \sum_{i=1}^{n} \bar{H}_{i}\left(\frac{x}{y}\right) \mathrm{d} G(y)+(1-\theta) \int_{0}^{\infty} \bar{F}\left(\frac{x}{y}\right) \mathrm{d} G(y) \\
& +\theta \int_{0}^{\infty} \bar{F}\left(\frac{x}{y}\right) \mathrm{d} G^{2}(y) \\
= & \sum_{i=2}^{n+1} \bar{H}_{i}(x)+\int_{0}^{\infty} \bar{F}\left(\frac{x}{y}\right) \mathrm{d} G_{\theta}(y) \\
& \sim \sum_{i=1}^{n+1} \bar{H}_{i}(x)
\end{aligned}
$$

showing that relation (4.3) still holds for $n+1$.

Finally, consider $(\mathrm{n}+1: 3)$. Note that $H_{n} \in \&$ by $(\mathrm{n}: 3)$ and that $\bar{G}(b(x))=o\left(\bar{H}_{n}(x)\right)$ and $\bar{H}_{n}(x-b(x)) \sim \bar{H}_{n}(x)$ by (n:1). Thus, applying Lemma 4.1 we obtain $H_{n+1} \in \&$. In other 
words, $H_{i} \in \& \subset \mathcal{L}$ for all $i=1, \ldots, n+1$. Hence, by (4.15), $T_{n+1}$ is long tailed. Moreover, by (4.15) again, if $\bar{G}(1)>0$ then $\operatorname{Pr}\left(T_{n+1}>x\right) \asymp \bar{H}_{n+1}(x)$, while if $\bar{G}(1)=0$ then $\operatorname{Pr}\left(T_{n+1}>x\right) \asymp \bar{H}(x)$. Therefore, for both cases, applying Theorem 2.1(a) of Klüppelberg (1988) we obtain the subexponentiality of $T_{n+1}$. This completes the proof of Theorem 3.1 .

\subsection{Proof of Theorem 3.2}

The following two lemmas are restatements of Lemma 3.2 and Theorem 2.1 of Tang (2006b), respectively.

Lemma 4.6. For two distribution functions $G$ and $H$ with $\bar{G}(x)>0$ and $\bar{H}(x)>0$ for all $x \in \mathbb{R}^{+}$, the relation $\bar{G}(c x)=o(\bar{H}(x))$ holds for all $c>0$ if and only if there is a function $b(\cdot): \mathbb{R}^{+} \mapsto \mathbb{R}^{+}$such that $(a)-(c)$, namely,

- $b(x) \uparrow \infty$,

- $b(x) / x \downarrow 0$, and

- $\bar{G}(b(x))=o(\bar{H}(x))$

hold.

Lemma 4.7. Let $X$ and $Y$ be two independent random variables with distribution functions $F$ on $\mathbb{R}$ and $G$ on $\mathbb{R}^{+}$, respectively. Denote by $H$ the distribution function of their product $X Y$. If $F \in \mathcal{A}$ and $\bar{G}(c x)=o(\bar{H}(x))$ for all $c>0$ then $H \in \mathcal{A}$.

Recall relations (4.8) and (4.9) under the conditions of Lemma 4.2. Thus, applying Lemma 4.7, we can easily obtain an analogue of Lemma 4.3 as follows.

Lemma 4.8. Let $(X, Y)$ follow a bivariate FGM distribution function (1.3) with $\theta \in(-1,1]$. If $F \in \mathcal{A}$ and $(a)-(c)$ hold, then $H \in \mathcal{A}$.

Proof of Theorem 3.2. As mentioned before, we only need to prove that relation (4.3) holds and that the distribution function of $T_{n}$ belongs to the class $\mathcal{A}$. We still proceed by induction on $n$. Note that

(1:1) $\bar{G}(b(x))=o(\bar{H}(x))$,

(1:2) relation (4.3) trivially holds for $n=1$,

(1:3) $T_{1}=X_{1} Y_{1}$ follows a distribution function in the class $\mathcal{A}$ (by Lemma 4.8).

Now assume that

(n:1) $\bar{G}(b(x))=o\left(\bar{H}_{n}(x)\right)$,

(n:2) relation (4.3) holds for $n$,

(n:3) both $X_{n} \prod_{j=1}^{n} Y_{j}$ and $T_{n}$ follow distribution functions in the class $\mathcal{A}$.

We will prove that these assertions hold for $n+1$, namely,

$(\mathrm{n}+1: 1) \bar{G}(b(x))=o\left(\bar{H}_{n+1}(x)\right)$,

$(\mathrm{n}+1: 2)$ relation (4.3) holds for $n+1$,

(n+1:3) both $X_{n+1} \prod_{j=1}^{n+1} Y_{j}$ and $T_{n+1}$ follow distribution functions in the class $\mathcal{A}$. 
The same as in the proof of Theorem 3.1, the relations in (4.13) hold. For $(n+1: 1)$, the verification of $\bar{G}(b(x))=o\left(\bar{H}_{n+1}(x)\right)$ is the same as in the proof of Theorem 3.1. For $(\mathrm{n}+1: 2)$, recalling Lemma 4.6, by relations (4.3) and (4.13) for $n$, the derivations of (4.14)-(4.15) are still valid, showing that relation (4.3) holds for $n+1$.

Finally, consider $(\mathrm{n}+1: 3)$. Note that $H_{n} \in \mathcal{A}$ by $(\mathrm{n}: 3)$ and that $\bar{G}(b(x))=o\left(\bar{H}_{n}(x)\right)$ by (n:1). Thus, applying Lemmas 4.6 and 4.7, we obtain $H_{n+1} \in \mathcal{A}$. In other words, $H_{i} \in \mathcal{A} \subset \&$ for all $i=1, \ldots, n+1$. Then, similarly as in the proof of Theorem 3.1, we obtain the subexponentiality of $T_{n+1}$. Furthermore, by definition, $H_{i} \in \mathcal{A}$ implies that there is some constant $c_{i}>1$ such that

$$
\limsup _{x \rightarrow \infty} \frac{\bar{H}_{i}\left(c_{i} x\right)}{\bar{H}_{i}(x)}<1 .
$$

With $c=\max \left\{c_{1}, c_{2}, \ldots, c_{n+1}\right\}>1$, by (4.15) we have

$$
\begin{aligned}
\limsup _{x \rightarrow \infty} \frac{\operatorname{Pr}\left(T_{n+1}>c x\right)}{\operatorname{Pr}\left(T_{n+1}>x\right)} & \leq \limsup _{x \rightarrow \infty} \frac{\sum_{i=1}^{n+1} \bar{H}_{i}\left(c_{i} x\right)}{\sum_{i=1}^{n+1} \bar{H}_{i}(x)} \\
& \leq \max _{1 \leq i \leq n+1} \limsup _{x \rightarrow \infty} \frac{\bar{H}_{i}\left(c_{i} x\right)}{\bar{H}_{i}(x)} \\
& <1 .
\end{aligned}
$$

Therefore, the distribution function of $T_{n+1}$ belongs to the class $\mathcal{A}$. This completes the proof of Theorem 3.2.

\subsection{Proof of Corollary 3.1}

Copied below is the well-known Breiman theorem; see Breiman (1965) and Cline and Samorodnitsky (1994).

Lemma 4.9. Let $X$ and $Y$ be two independent random variables with distribution functions $F$ on $\mathbb{R}$ and $G$ on $\mathbb{R}^{+}$, respectively. Denote by $H$ the distribution function of their product $X Y$. If $F \in \operatorname{MDA}\left(\Phi_{\alpha}\right)$ for some $\alpha>0$ and $\mathrm{E}\left[Y^{\alpha+\varepsilon}\right]<\infty$ for some $\varepsilon>0$, then

$$
\lim _{x \rightarrow \infty} \frac{\bar{H}(x)}{\bar{F}(x)}=\mathrm{E}\left[Y^{\alpha}\right]
$$

The following lemma forms the main ingredient of the proof of Corollary 3.1.

Lemma 4.10. The following statements hold.

(i) Under the conditions of Corollary 3.1(i), it holds for each $i \in \mathbb{N}$ that

$$
\bar{H}_{i}(x) \sim\left((1-\theta) \mathrm{E}\left[Y^{\alpha}\right]+\theta \mathrm{E}\left[\left(Y_{1}^{*} \vee Y_{2}^{*}\right)^{\alpha}\right]\right)\left(\mathrm{E}\left[Y^{\alpha}\right]\right)^{i-1} \bar{F}(x),
$$

where $Y_{1}^{*}$ and $Y_{2}^{*}$ are two independent random variables identically distributed as $Y$.

(ii) Under the conditions of Corollary 3.1(ii), it holds for each $i \in \mathbb{N}$ that

$$
\bar{H}_{i}(x) \sim(1+\theta) \bar{F}\left(\frac{x}{\hat{y}^{i}}\right)\left(\Gamma(\alpha+1) \bar{G}\left(\hat{y}-\frac{\hat{y}^{i+1}}{x} a\left(\frac{x}{\hat{y}^{i}}\right)\right)\right)^{i} .
$$


Proof. (i) Apply Lemma 4.9 to relation (4.4) to obtain

$$
\lim _{x \rightarrow \infty} \frac{\bar{H}(x)}{\bar{F}(x)}=(1-\theta) \mathrm{E}\left[Y^{\alpha}\right]+\theta \mathrm{E}\left[\left(Y_{1}^{*} \vee Y_{2}^{*}\right)^{\alpha}\right] ;
$$

see also Theorem 2.1(i) of Jiang and Tang (2011) for a result more general than (4.16). Thus, the distribution function $H$ of $X_{i} Y_{i}$ belongs to the class $\operatorname{MDA}\left(\Phi_{\alpha}\right)$. Then, for $i=2,3, \ldots$, by Lemmas 4.9 and relation (4.16), in turn,

$$
\begin{aligned}
\bar{H}_{i}(x) & =\operatorname{Pr}\left(X_{i} Y_{i} \prod_{j=1}^{i-1} Y_{j}>x\right) \\
& \sim\left(\mathrm{E}\left[Y^{\alpha}\right]\right)^{i-1} \bar{H}(x) \\
& \sim\left((1-\theta) \mathrm{E}\left[Y^{\alpha}\right]+\theta \mathrm{E}\left[\left(Y_{1}^{*} \vee Y_{2}^{*}\right)^{\alpha}\right]\right)\left(\mathrm{E}\left[Y^{\alpha}\right]\right)^{i-1} \bar{F}(x) .
\end{aligned}
$$

(ii) Note that every distribution function $F \in \operatorname{MDA}(\Lambda)$ with an upper endpoint $\hat{x}=\infty$ is rapidly varying tailed. We apply Theorem 2.1(ii) of Jiang and Tang (2011), which, in our situation, implies that

$$
\lim _{x \rightarrow \infty} \frac{\operatorname{Pr}(X Y>x)}{\operatorname{Pr}\left(X^{*} Y^{*}>x\right)}=1+\theta,
$$

where $X^{*}$ and $Y^{*}$ are two independent random variables with $X^{*}$ identically distributed as $X$ and $Y^{*}$ as $Y$, and are independent of $\left\{\left(X_{n}, Y_{n}\right), n \in \mathbb{N}\right\}$. Then, by (4.17) and Theorem 3.1(i) of Hashorva et al. (2010), for $i=1,2, \ldots$,

$$
\begin{aligned}
\bar{H}_{i}(x) & =\mathrm{E}\left[\operatorname{Pr}\left(X_{i} Y_{i} \prod_{j=1}^{i-1} Y_{j}>x \mid Y_{1}, \ldots, Y_{i-1}\right)\right] \\
& \sim(1+\theta) \operatorname{Pr}\left(X^{*} Y^{*} \prod_{j=1}^{i-1} Y_{j}>x\right) \\
& =(1+\theta) \operatorname{Pr}\left(X^{*} \prod_{j=1}^{i} \frac{Y_{j}}{\hat{y}}>\frac{x}{\hat{y}^{i}}\right) \\
& =(1+\theta) \bar{F}\left(\frac{x}{\hat{y}^{i}}\right)\left(\Gamma(\alpha+1) \bar{G}\left(\hat{y}-\frac{\hat{y}^{i+1}}{x} a\left(\frac{x}{\hat{y}^{i}}\right)\right)\right)^{i} .
\end{aligned}
$$

This completes the proof of Lemma 4.10.

Proof of Corollary 3.1. For statement (i), by (4.16), it is easy to verify that (a)-(c) hold with $b(x)=x^{1-\delta}$ for some $0<\delta<\varepsilon /(\alpha+\varepsilon)$; see also Lemma 3.7 of Tang and Tsitsiashvili (2003). For statement (ii), (a)-(c) automatically hold because $Y$ is bounded above. Hence, Theorem 3.2 is applicable for both statements (i) and (ii). Then we apply Lemma 4.10 to (3.1) to complete the proof of Corollary 3.1.

\section{Acknowledgements}

The author is most grateful to Dr. Yang Yang and an anonymous referee for their very helpful comments, which have helped to significantly improve the presentation of this paper. The author also acknowledges support from the National Natural Science Foundation of China (grant number 70871104) for research travel during this project. 


\section{References}

Breiman, L. (1965). On some limit theorems similar to the arc-sin law. Theory Prob. Appl. 10, 323-331.

Cline, D. B. H. (1986). Convolution tails, product tails and domains of attraction. Prob. Theory Relat. Fields $\mathbf{7 2 ,}$ $529-557$.

Cline, D. B. H. And SAmorodnitsky, G. (1994). Subexponentiality of the product of independent random variables. Stoch. Process. Appl. 49, 75-98.

Cossette, H., Marceau, E. ANd Marri, F. (2008). On the compound Poisson risk model with dependence based on a generalized Farlie-Gumbel-Morgenstern copula. Insurance Math. Econom. 43, 444-455.

Embrechts, P. AND Goldie, C. M. (1980). On closure and factorization properties of subexponential and related distributions. J. Austral. Math. Soc. Ser. A 29, 243-256.

Embrechts, P., Klüppelberg, C. And Mikosch, T. (1997). Modelling Extremal Events. Springer, Berlin.

Goovaerts, M. J. et al. (2005). The tail probability of discounted sums of Pareto-like losses in insurance. Scand. Actuarial J. 2005, 446-461.

Grey, D. R. (1994). Regular variation in the tail behaviour of solutions of random difference equations. Ann. Appl. Prob. 4, 169-183.

Hashorva, E., Pakes, A. G. ANd TAng, Q. (2010). Asymptotics of random contractions. Insurance Math. Econom. 47, 405-414.

JIANG, J. AND TANG, Q. (2011). The product of two dependent random variables with regularly varying or rapidly varying tails. Statist. Prob. Lett. 81, 957-961.

KLÜPPELberG, C. (1988). Subexponential distributions and integrated tails. J. Appl. Prob. 25, 132-141.

Konstantinides, D., TAng, Q. And Tsitsiashvili, G. (2002). Estimates for the ruin probability in the classical risk model with constant interest force in the presence of heavy tails. Insurance Math. Econom. 31, 447-460.

Kotz, S., Balakrishnan, N. and Johnson, N. L. (2000). Continuous Multivariate Distributions, Vol. 1, 2 nd edn. Wiley-Interscience, New York.

Nyrhinen, H. (1999). On the ruin probabilities in a general economic environment. Stoch. Process. Appl. 83, 319-330.

Nyrhinen, H. (2001). Finite and infinite time ruin probabilities in a stochastic economic environment. Stoch. Process. Appl. 92, 265-285.

TANG, Q. (2006a). Asymptotic ruin probabilities in finite horizon with subexponential losses and associated discount factors. Prob. Eng. Inf. Sci. 20, 103-113.

TANG, Q. (2006b). The subexponentiality of products revisited. Extremes 9, 231-241.

Tang, Q. and Tsitsiashvili, G. (2003). Precise estimates for the ruin probability in finite horizon in a discrete-time model with heavy-tailed insurance and financial risks. Stoch. Process. Appl. 108, 299-325.

TANG, Q. AND Tsitsiashvili, G. (2004). Finite- and infinite-time ruin probabilities in the presence of stochastic returns on investments. Adv. Appl. Prob. 36, 1278-1299.

TANG, Q. AND Vernic, R. (2007). The impact on ruin probabilities of the association structure among financial risks. Statist. Prob. Lett. 77, 1522-1525.

Teugels, J. L. (1975). The class of subexponential distributions. Ann. Prob. 3, 1000-1011.

WENG, C., ZHANG, Y. AND TAN, K. S. (2009). Ruin probabilities in a discrete time risk model with dependent risks of heavy tail. Scand. Actuarial J. 2009, 205-218.

Yi, L., Chen, Y. AND Su, C. (2011). Approximation of the tail probability of randomly weighted sums of dependent random variables with dominated variation. J. Math. Anal. Appl. 376, 365-372.

Zhang, Y., Shen, X. And Weng, C. (2009). Approximation of the tail probability of randomly weighted sums and applications. Stoch. Process. Appl. 119, 655-675.

Zhou, M., WANG, K. AND WANG, Y. (2011). Estimates for the finite-time ruin probability with insurance and financial risks. To appear in Acta Math. Appl. Sinica. 